\title{
Electronic properties of complex oxide interfaces
}

\author{
M. Varela*, H. M. Christen*, H.N. Lee*, S.J. Pennycook*. \\ *Condensed Matter Sciences Division, Oak Ridge National Laboratory, Oak Ridge, TN \\ 37831 USA
}

\section{Peña**, Z. Sefrioui**, J. Santamaria** \\ **GFMC. Dpto de Fisica Aplicada III. Universidad Complutense. 28040 Madrid, Spain.}

Complex oxides with perovskite-based structures exhibit a number of different ground states including ferromagnetic, superconducting, metallic, insulating or ferroelectric phases, which are extremely sensitive to doping. In particular, complex oxide interfaces where the bonding is disturbed can present quite unusual physical properties due to the presence of charge transfer, localization or unusual electronic reconstructions [1-2]. Harnessing such phenomena relies on probes capable to provide a comprehensive structural, chemical and electronic characterization at the atomic scale.

Atomic resolution electron energy loss spectroscopy (EELS) in the aberration corrected scanning transmission electron microscope (STEM) is a very powerful tool with sensitivity to identify individual atoms [4] within their bulk environment and determine their local electronic structure. Thus, the combination of STEM-EELS is unique when it comes to mapping the structure, chemistry and properties of complex oxide interfaces with sub-Ångstrom resolution. For example, the ability to probe charge transfer at the atomic level uncovers substantial changes in properties in the regions close to ferromagnetic/superconducting ( $\mathrm{F} / \mathrm{S}) \mathrm{YBa}_{2} \mathrm{Cu}_{3} \mathrm{O}_{7-\mathrm{x}} / \mathrm{La}_{0.7} \mathrm{Ca}_{0.3} \mathrm{MnO}_{3} \quad(\mathrm{YBCO} / \mathrm{LCMO})$ interfaces, leading to a microscopic explanation of macroscopic observations. Figure 1 shows the fingerprint of significant charge transfer taking place in a $\mathrm{F} / \mathrm{S} \mathrm{YBCO} / \mathrm{LCMO}$ heterostructure. These results have been obtained from the analysis of the fine structure of independent linescans of the $\mathrm{O} \mathrm{K}$ edge at $530 \mathrm{eV}$ (the pre-peak intensity was analyzed for YBCO) and the Mn L edge at $644 \mathrm{eV}$ (the Mn oxidation state was inferred from $\mathrm{L}_{23}$ ratios in LCMO). In the superconductor, the hole density is strongly depressed within $3 \mathrm{~nm}$ of the interface, while the hole density in the ferromagnet is correspondingly enhanced. The critical temperatures $(\mathrm{Tc})$ of these multilayers are lower than YBCO-based superlattices with $\mathrm{PrBa}_{2} \mathrm{Cu}_{3} \mathrm{O}_{7}$ (PBCO) instead, where no charge transfer has been observed. Therefore, such effects should be taken into account when explaining the properties of thin layers in these $\mathrm{F} / \mathrm{S}$ superlattices.

A rather different behavior arises in $\mathrm{LaMnO}_{3} / \mathrm{SrTiO}_{3}$ superlattices. The interfaces in this system show two different structures: ...- $\mathrm{MnO}_{2}-\mathrm{LaO}-\mathrm{TiO}_{2}-\mathrm{SrO}_{2}-$. versus ...-LaO$\mathrm{MnO}_{2}-\mathrm{SrO}-\mathrm{TiO}_{2}-\ldots$ as shown in figure 2, where the elemental profiles of $\mathrm{La}, \mathrm{Mn}$ and $\mathrm{Ti}$ are shown across the superlatttice stacking show clearly the two types of terminations. Interestingly, at these interfaces the oxidation state changes towards increasing the $\mathrm{Mn}$ valence (inferred from measurements of $\mathrm{L}_{23}$ ratios) but the change is different for each of the observed structures. Meanwhile, the Ti oxidation state remains constant suggesting that a different mechanism to charge transfer is taking place.

[1] A. Ohtomo and H. Y. Hwang, Nature 427, (2004) 423

[2] S. Okamoto and A. J. Millis, Nature 428, (2004) 630

[3] M. Varela et al., Phys. Rev. Lett. 92, (2004) 095502

[4] Z. Sefrioui et al., Appl. Phys. Lett. 81, (2002) 4568 
[5] N. Browning et al., Physica C 202, (1992) 12

[6] This work was supported by DOE BES Division of Materials Sciences under contract DE-AC05-00OR22725 managed by UT-Battelle, LLC.
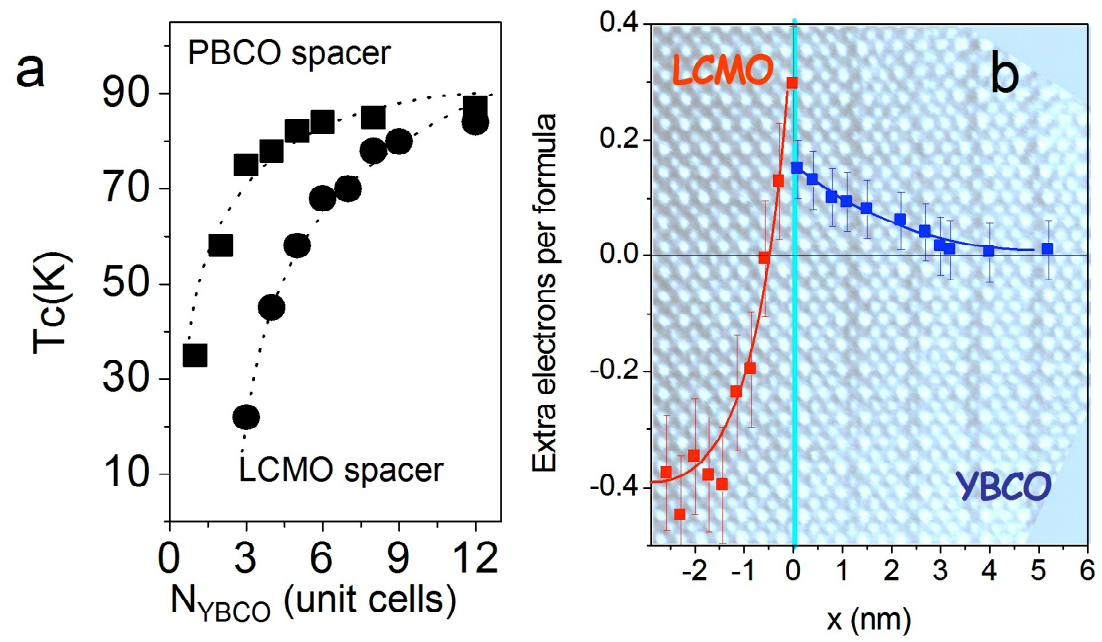

Figure 1(a) Dependence of the Tc on the superconducting layer thickness for a set of YBCO/LCMO superlattices with a fixed LCMO thickness of $6 \mathrm{~nm}$ (circles). Squares represent the Tc of a similar set of samples with an insulating PBCO spacer (data from reference 5). (b) Excess electrons per formula per unit cell across the $\mathrm{YBCO}$ (blue)/ LCMO (red) interface. EEL spectra from the parent compounds $\mathrm{LaMnO}_{3}$ and $\mathrm{CaMnO}_{3}$ and data from ref [5] were used as an internal calibration. The image in the background is a Z-contrast image in real scale and is only a guide to the eye (it was not acquired simultaneously with EELS data). The interface position has been marked with a vertical line, while the bulk doping level (zero in this representation) has been highlighted with a horizontal line.
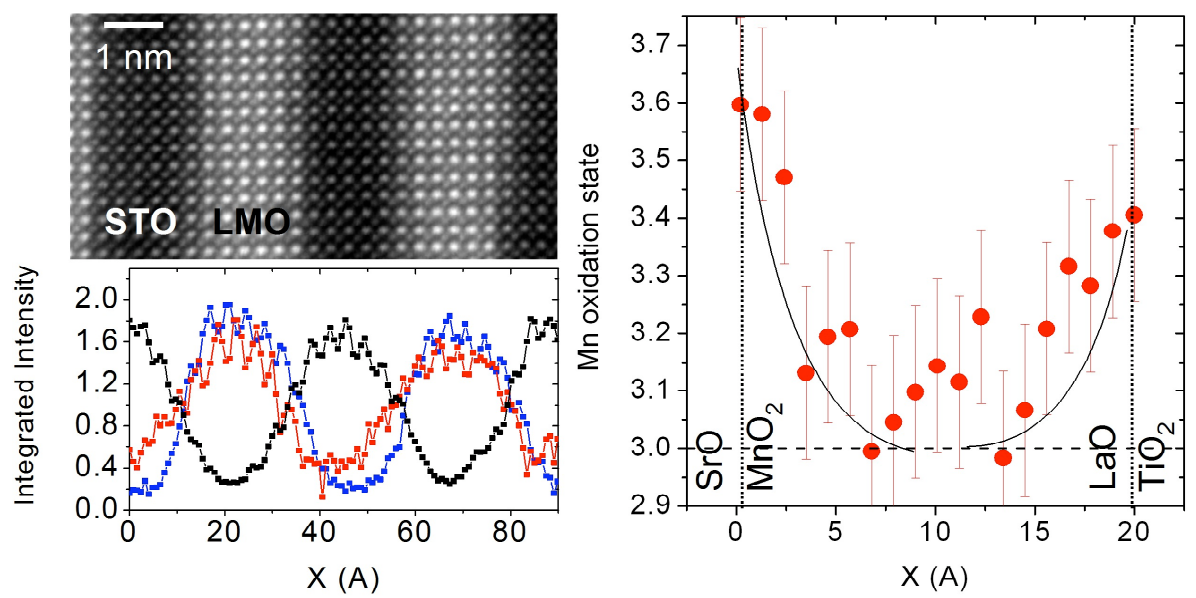

Figure 2: (Top left) Z-contrast image of a LMO/STO superlattice. (Bottom left) Elemental concentrations of Ti (black), Mn (red) and La (blue) across the stacking of two bilayers. Concentrations are represented by the integrated intensities under the $\mathrm{Ti}$ $\mathrm{L}, \mathrm{Mn} \mathrm{L}$ and La M edges respectively. (Right) Mn oxidation state, inferred from the $\mathrm{L}_{23}$ ratio across a LMO layer (the parent compounds $\mathrm{LaMnO}_{3}$ and $\mathrm{CaMnO}_{3}$ were used as an internal calibration). 